\title{
Electro-osmotic flow of a third-grade fluid past a channel having stretching walls
}

https://doi.org/10.1515/nleng-2017-0112

Received August 31, 2017; revised February 5, 2018; accepted March 17, 2018.

\begin{abstract}
The electro-osmotic flow of a third grade fluid past a channel having stretching walls has been studied in this paper. The channel height is taken much greater than the thickness of the electric double layer comprising of the Stern and diffuse layers. The equations governing the flow are obtained from continuity equation, the Cauchy's momentum equation and the Poisson-Boltzmann equation. The Debye-Hückel approximation is adopted to linearize the Poisson-Boltzmann equation. Suitable similarity transformations are used to reduce the resulting nonlinear partial differential equation to ordinary differential equation. The reduced equation is solved numerically using damped Newton's method. The results computed are presented in form of graphs.
\end{abstract}

Keywords: Electro-osmosis; Damped Newton's method; Third-grade fluid; Coefficient of skin-friction

MSC: 76A05, 76W05, 76M20

\section{Introduction}

The electroosmotic flow (EOF) is the flow induced by the application of an electric field across a channel and due to the presence of electric double layer (EDL) at channel walls. Electric double layers exist in all heterogeneous fluid based systems including biofluids. The electroosmotic flow is most significant in small channels where the EDL is much smaller than the characteristic length scale of the channel. The electroosmotic flow is considered to be an inseparable part of many scientific and engineering applications used in microfluidic devices. The various applications of microfluidic devices include micro-reactors,

\footnotetext{
*Corresponding Author: Mamata Parida, Department of Mathematics, Utkal University, Bhubaneswar 751 004, India, E-mail: mamatakmru@gmail.com

Sudarsan Padhy, Department of Mathematics, Utkal University, Bhubaneswar 751 004, India
}

heat transfer in electronic circuits, biosensors, drug delivery, DNA analysis and sequencing, etc. It has been projected that microfluidic devices utilizing EOF will have great application in medical research $[1,2]$. As the biofluids are electrically conducting in nature, EOF will be vital for drug delivery and separation/mixing of fluids at the atomic level. Studies on EOF of Newtonian fluids abound in contrast to non-Newtonian fluids as evident from the literature. In the recent years, to handle complex fluids in microfluidics, EOF of non-Newtonian fluids has gained attention.

The study of EOF was first reported by F.F. Reuss [3] in 1809 where he has showed that water can flow through a plug of clay by the application of suitable electric voltage. Most of the early studies [4-6] have focused on the theoretical analysis of electroosmotic flow of Newtonian fluids. Santiago [7] analyzed the effects of fluid inertia and pressure on the velocity and vorticity field of electroosmotic flow of Newtonian fluids. The analytical solution for electrostatic potential of double layer has been obtained by solving the complete Poisson-Boltzmann equation for arbitrary zeta potentials using Green's function method for Newtonian fluids in [8]. A theoretical model was developed by Chakraborty [9] for studying the capillary filling dynamics of a power-law fluid in a microchannel. Park and Lee [10] numerically obtained flow field solution of full Phan-Thien and Tanner (PTT) constitutive equation in a rectangular duct under the action of an external electric field and applied pressure gradient by employing finite volume method. Berli and Olivares [11] introduced a theoretical description of the electrokinetic flow of power law fluid through slit and cylindrical microchannels. Exact solution of the velocity distribution are obtained for the EOF of power-law fluids in a slit channel analytically by Zhao et al. [12]. Siddiqui and Lakhatia [13] fomulated and solved the boundary value problem of steady, symmetric one-dimensional electroosmotic flow of a micropolar fluid in a uniform rectangular microchannel under the action of uniformly applied electric field. A numerical study of electroosmotic flow in microchannels for non-Newtonian fluid obeying power-law model was attempted for the first time in [14]. Analytical 
solutions for channel and pipe flows of visco-elastic fluids obeying PTT model and the FENE-P model, under the mixed influence of electrokinetic and pressure forces were obtained in [15]. Vasu and De [16] have numerically analyzed the fluid flow behavior for EOF of power-law fluids in a rectangular microchannel at high zeta potential by considering the EDL potential distribution without Debye Huckel linear approximation. A numerical investigation of flow of power-law fluid in a two dimensional microchannel with non-uniform zeta potential distributions along the channel walls by finite volume scheme was carried out by Hadigol et al. [17]. Analytical solutions were obtained for transverse distribution of velocity, and thermal transport characteristics of the steady fully developed electroosmotic flow of PTT and FENE-P model fluid in a slit microchannel in [18]. Semi-analytical solutions for transient EOF of Maxwell fluids between micro-parallel plates are presented in [19] using Laplace transform method. A numerical analysis is performed in [20] on alternating current EOF of non-Newtonian incompressible fluids based on the Ostwald-de Waele power-law model in circular microchannels. The electroosmotic flow of Maxwell fluids in a rectangular microchannel with asymmetric high zeta potentials at the walls is studied analytically using Green's functions and separation of variables method be Jimenez et al. [21]. Xie and Jian [22] analyzed the rotating electroosmotic flow of power-law fluids at high zeta potentials in a slit microchannel using finite difference method. Electroosmotic flow through a channel between two undulated surfaces induced by an external electric field is studied in [23] using lubrication approximation theory and lattice Boltzmann method. The EOF of power-law fluids in microchannels with different geometries is discussed in [24, 25].

A theoretical study on the electroosmotic flow of a fluid past a channel having stretching walls has been investigated by Misra et al. [26] by considering the biofluid to be a second grade fluid. Although the second grade fluid model can predict the characteristics of biofluids, it does not take into account the shear thinning phenomenon which is a vital property of biofluids. This motivated us to extend their work and analyze the electroosmotic flow of a third grade fluid past a channel having stretching walls in the present work. The partial differential equation governing the flow is transformed to a nonlinear ordinary differential equation using appropriate similarity transformation which is then solved using a suitable finite difference scheme. The results computed are presented in the form of graphs for various flow parameters governing the flow.

\section{Basic Equations}

The basic laws of the conservation of mass, conservation of momentum, and conservation of energy for an incompressible fluid are

$$
\begin{aligned}
& \nabla \cdot \mathbf{V}=0, \\
& \rho \frac{D \mathbf{V}}{D t}=\nabla \cdot \boldsymbol{\tau}+\mathbf{F}, \\
& \rho C_{p} \frac{D T}{D t}=\kappa \nabla^{2} T+\boldsymbol{\tau} \cdot(\nabla \mathbf{V}),
\end{aligned}
$$

where $\mathbf{V}$ is the velocity field, $\rho$ is the fluid density, $C_{p}$ is the specific heat at constant pressure, $\kappa$ is the coefficient of thermal conductivity, $\mathbf{F}$ is the body force per unit volume, $T$ is the temperature, and $\boldsymbol{\tau}$ is the stress tensor. The constitutive equation of Cauchy stress tensor for a third grade fluid is

$$
\begin{aligned}
\boldsymbol{\tau}= & -p \mathbf{I}+\mu \mathbf{A}_{1}+\alpha_{1} \mathbf{A}_{2}+\alpha_{2} \mathbf{A}_{1}^{2}+\beta_{1} \mathbf{A}_{3} \\
& +\beta_{2}\left(\mathbf{A}_{1} \mathbf{A}_{2}+\mathbf{A}_{2} \mathbf{A}_{1}\right)+\beta_{3}\left(\operatorname{tr} \mathbf{A}_{1}^{2}\right) \mathbf{A}_{1},
\end{aligned}
$$

where $p$ is the pressure, $\mu$ is the coefficient of viscosity, $\alpha_{1}, \alpha_{2}, \beta_{1}, \beta_{2}, \beta_{3}$ are material constants and the RivlinEricksen tensors $\mathbf{A}_{1}, \mathbf{A}_{2}$, and $\mathbf{A}_{3}$ are given by

$$
\begin{aligned}
& \mathbf{A}_{1}=\nabla \mathbf{V}+(\nabla \mathbf{V})^{\prime}, \\
& \mathbf{A}_{n}=\frac{D \mathbf{A}_{n-1}}{D t}+\mathbf{A}_{n-1}(\nabla \mathbf{V})+(\nabla \mathbf{V})^{\prime} \mathbf{A}_{n-1} \text { for } n \geq 2 .
\end{aligned}
$$

Fosdick and Rajagopal [27] have shown that the motion satisfies Clausius-Duhem inequality to be compatible with thermodynamics and if it is assumed that the specific Helmholtz free energy is minimum when the fluid is at rest then the following constraints must be satisfied

$\mu \geq 0, \alpha_{1} \geq 0,\left|\alpha_{1}+\alpha_{2}\right| \leq \sqrt{24 \mu \beta_{3}}, \beta_{1}=\beta_{2}=0, \beta_{3} \geq 0$.

The $\mathbf{F}$ term in equation (2) is given as

$$
\mathbf{F}=\rho_{e} \mathbf{E},
$$

where $\mathbf{E}$ is the applied electric field and $\rho_{e}$ is the net electric charge density. For a symmetric electrolyte the anions and cations have same charge valence $z^{+}=z^{-}=z$ and the net electric charge density $\rho_{e}=e z\left(n^{+}-n^{-}\right)$where $n^{+}$and $n^{-}$are the concentrations of positive and negative ions, respectively and e is the elementary electric charge.

The electric field intensity $\mathbf{E}=-\nabla \Phi$, where $\Phi$ is the electric potential governed by

$$
\nabla^{2} \Phi=-\frac{\rho_{e}}{\varepsilon},
$$

where $\varepsilon$ is the electric permittivity of the solution. In electroosmotic flows, two types of electric fields act on 
the flow. One is the applied electric field $\phi$ which is generated by the electrodes at the inlet and outlet of the channel and the other one $\psi$ is due to the net charge distribution in the electric double layer which is acquired at the wall. The total electric field $\Phi$ is a linear superposition of $\phi$ and $\psi$.

$$
\begin{aligned}
\nabla^{2} \phi & =0, \\
\nabla^{2} \psi & =-\frac{\rho_{e}}{\varepsilon} .
\end{aligned}
$$

At the centerline of the channel the EDL does not overlap and variation of the electrical potential distribution $\psi$ occurs only in the normal direction to the channel walls which can be expressed in the $y$-direction as

$$
\frac{d^{2} \psi}{d y^{2}}=-\frac{\rho_{e}}{\varepsilon} .
$$

According to Bruss [28], the net electric charge density in the EDL can be expressed as

$$
\rho_{e}=-2 z e n_{0} \sinh \left(\frac{z e \psi}{k_{B} T_{A}}\right),
$$

where $n_{0}$ is the bulk number concentration of ions in the electrolyte solution, $k_{B}$ is the Boltzmann constant and $T_{A}$ is the absolute temperature. The Debye-Hückel linear approximation yields

$$
\sinh (.) \equiv(.)
$$

\section{Problem Formulation}

We consider the fully developed electroosmotic flow of a viscous incompressible fluid, that obeys the third grade fluid model, in a channel bounded by stretching walls with equations $y= \pm R_{0}$ (Fig.1). The flow is assumed to be symmetric about the centerline and hence we confine our study to the region $0 \leq y \leq R_{0}$. The height of the channel is much smaller than the length of the channel, $L$. The effect of Joule heating, gravity, and pressure gradient have been neglected in the present study. The flow is driven by stretching of the channel walls and the electro-osmotic effect. The channel walls in contact with the electrolyte develop surface charge due to ion adsorption which results in the formation of the electric double layer. The physical extent of the EDL is represented by the Debye length. The EDL is very thin, therefore the effective fluid velocity is quantified by the Helmholtz-Smoluchowski equation

$$
U_{H S}=-\frac{\varepsilon \zeta E_{\chi}}{\mu},
$$

where $\zeta$ is the zeta potential. In the boundary layer, the equations that govern the flow for the present problem are [29]

$$
\frac{\partial u}{\partial x}+\frac{\partial v}{\partial y}=0
$$

$$
\text { and } \begin{aligned}
& \rho\left(u \frac{\partial u}{\partial x}+v \frac{\partial u}{\partial y}\right)=\mu \frac{\partial^{2} u}{\partial y^{2}} \\
& +\alpha_{1}\left[u \frac{\partial^{3} u}{\partial x \partial y^{2}}+\frac{\partial u}{\partial x} \frac{\partial^{2} u}{\partial y^{2}}+3 \frac{\partial u}{\partial y} \frac{\partial^{2} v}{\partial y^{2}}+v \frac{\partial^{3} u}{\partial y^{3}}\right] \\
& +2 \alpha_{2} \frac{\partial u}{\partial y} \frac{\partial^{2} v}{\partial y^{2}}+6 \beta_{3}\left(\frac{\partial u}{\partial y}\right)^{2} \frac{\partial^{2} u}{\partial y^{2}}+\rho_{e} E_{x} .
\end{aligned}
$$

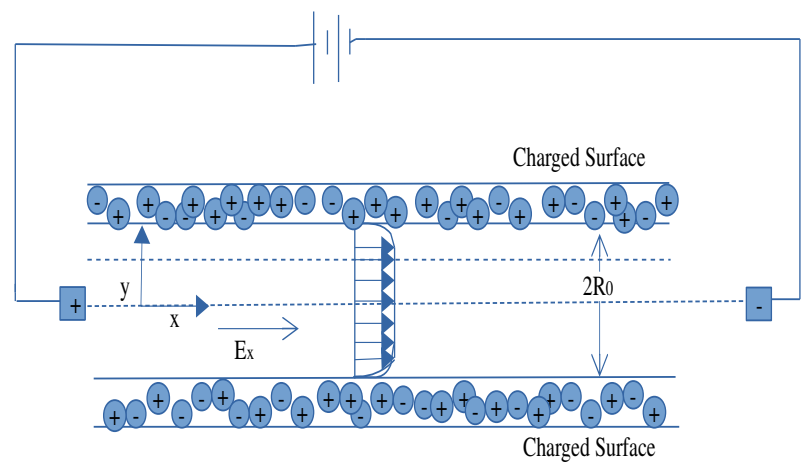

Fig. 1: Sketch of the problem

The electric potential distribution for a symmetric electrolyte described by the Poisson-Boltzmann equation in Eq.(5) and Eq.(6) is linearized using Eq.(7), and is given by

$$
\frac{d^{2} \psi}{d y^{2}}=\frac{\psi}{\lambda_{D}^{2}}
$$

where $\quad \lambda_{D}=\sqrt{\frac{\varepsilon k_{B} T_{A}}{2 n_{0} z^{2} e^{2}}} \quad$ is the Debye length.

The boundary conditions for the velocities and electric potential for the flow problem are

$$
\begin{array}{lll}
\text { At } & y=0: & \frac{\partial u}{\partial y}=0, \quad v=0, \quad \frac{d \psi}{d y}=0 . \quad(12) \\
\text { At } \quad y=R_{0}: & u=b x(b>0), \quad v=0, \quad \psi=\zeta .
\end{array}
$$

Using the following similarity transformation

$$
u=\frac{U_{H S} \chi f^{\prime}(\eta)}{R_{0}}, \quad v=-U_{H S} f(\eta)
$$

and introducing the non-dimensional variables

$$
x^{\star}=\frac{x}{R_{0}}, \quad \eta=\frac{y}{R_{0}}, \quad u^{*}=\frac{u}{U_{H S}}, \quad v^{\star}=\frac{v}{U_{H S}},
$$




$$
\psi^{\star}=\frac{\psi}{\zeta},
$$

the partial differential equation (10) transforms to the following ordinary differential equation

$$
\begin{aligned}
& \frac{1}{\operatorname{Re}}\left[f^{\prime 2}(\eta)-f(\eta) f^{\prime \prime}(\eta)\right]=f^{\prime \prime \prime}(\eta) \\
& +\alpha\left[2 f^{\prime}(\eta) f^{\prime \prime \prime}(\eta)-f(\eta) f^{(i v)}(\eta)-3 f^{\prime \prime 2}(\eta)\right] \\
& -2 y f^{\prime \prime 2}(\eta)+6 \beta \Lambda^{2} f^{\prime \prime 2}(\eta) f^{\prime \prime \prime}(\eta)+\Lambda \frac{d^{2} \psi^{\star}}{d \eta^{2}},
\end{aligned}
$$

where

$$
\alpha=\frac{\alpha_{1} U_{H S}}{R_{0} \mu}, y=\frac{\alpha_{2} U_{H S}}{R_{0} \mu}, \quad \beta=\frac{\beta_{3} U_{H S}^{2}}{\mu R_{0}^{2}},
$$

$R e=\frac{\rho U_{H S} R_{0}}{\mu}$, and $\Lambda=\frac{R_{0}}{L}$ is the aspect ratio.

The boundary conditions become

$$
\begin{array}{rrrl}
f(0)=0, & f^{\prime \prime}(0)=0, & \left.\frac{d \psi^{\star}}{d \eta}\right|_{\eta=0}=0, \\
f(1)=0, & f^{\prime}(1)=1, & \psi^{\star}(1)=1 .
\end{array}
$$

On solving the linearized Poisson-Boltzmann equation with the boundary conditions, we get

$$
\frac{d^{2} \psi^{\star}}{d \eta^{2}}=K^{2} \frac{\cosh K \eta}{\cosh K}, \quad K=\frac{R_{0}}{\lambda_{D}} .
$$

Eq. (13) now becomes

$$
\begin{aligned}
& \frac{1}{\operatorname{Re}}\left[f^{\prime 2}(\eta)-f(\eta) f^{\prime \prime}(\eta)\right]=f^{\prime \prime \prime}(\eta) \\
& +\alpha\left[2 f^{\prime}(\eta) f^{\prime \prime \prime}(\eta)-f(\eta) f^{(i v)}(\eta)-3 f^{\prime \prime 2}(\eta)\right] \\
& -2 y f^{\prime \prime 2}(\eta)++6 \beta \Lambda^{2} f^{\prime \prime 2}(\eta) f^{\prime \prime \prime}(\eta) \\
& +\Lambda K^{2} \frac{\cosh K \eta}{\cosh K}
\end{aligned}
$$

\section{Numerical Methods}

The resulting equation arising from the problem of EOF of a third grade fluid past a channel having stretching walls is highly non-linear in nature and does not admit an exact analytical solution. We, therefore, look for a numerical solution of the problem under consideration. For simplicity, better stability, accuracy and efficiency, the finite difference technique is used for discretization. In order to discretize the differential quotients the following suitable finite difference strategy is opted.

We have divided the domain $[0,1]$ into a set of nonoverlapping grids such that the spacing of the grid points,
$\Delta \eta$ is uniform. The grid points are identified by an index $i$ running in the $\eta$-direction.

$$
\begin{array}{ll}
\eta_{i}=i \Delta \eta, & i=1,2, \ldots, N \\
f_{i} & =f\left(\eta_{i}\right) .
\end{array}
$$

At the initial point $(i=0)$, Euler's forward finite difference scheme and at the end point $(i=N)$, the backward finite difference is used.

For $i=1$, the following difference scheme is used

$$
\begin{aligned}
f_{i}^{\prime} & =\frac{f_{i+1}-f_{i-1}}{2 \Delta \eta}, \\
f_{i}^{\prime \prime} & =\frac{f_{i+1}-2 f_{i}+f_{i-1}}{(\Delta \eta)^{2}}, \\
f_{i}^{\prime \prime \prime} & =\frac{f_{i+2}-3 f_{i+1}+3 f_{i}-f_{i-1}}{(\Delta \eta)^{3}}, \\
f_{i}^{(i v)} & =\frac{f_{i+3}-4 f_{i+2}+6 f_{i+1}-4 f_{i}+f_{i-1}}{(\Delta \eta)^{4}} .
\end{aligned}
$$

The central difference scheme is used for discretization of the terms at the nodes $i=2,3, \ldots, N-2$.

For $i=N-1$, the following difference scheme is used

$$
\begin{aligned}
f_{i}^{\prime} & =\frac{f_{i+1}-f_{i-1}}{2 \Delta \eta}, \\
f_{i}^{\prime \prime} & =\frac{f_{i+1}-2 f_{i}+f_{i-1}}{(\Delta \eta)^{2}}, \\
f_{i}^{\prime \prime \prime} & =\frac{f_{i+1}-3 f_{i}+3 f_{i-1}-f_{i-2}}{(\Delta \eta)^{3}}, \\
f_{i}^{(i v)} & =\frac{f_{i+1}-4 f_{i}+6 f_{i-1}-4 f_{i-2}+f_{i-3}}{(\Delta \eta)^{4}} .
\end{aligned}
$$

The resulting algebraic system of equations is then expressed in the residual form as

$$
\mathcal{R}_{i}=0 \quad i=1,2, \ldots, N .
$$

where the residuals are given as follows

$$
\begin{aligned}
\mathcal{R}_{1} & =\frac{-1}{4 R e}\left(\frac{f_{2}}{\Delta \eta}\right)^{2}+\frac{2 f_{3}-3 f_{2}}{2(\Delta \eta)^{3}}-\frac{\alpha f_{2}}{2(\Delta \eta)^{4}}\left(f_{4}-6 f_{3}+7 f_{2}\right) \\
& +\Lambda K^{2} \frac{\cosh K \eta_{1}}{\cosh K} .
\end{aligned}
$$

For $\mathrm{m}=2,3, \ldots, \mathrm{N}-2$

$$
\begin{aligned}
\mathcal{R}_{m}= & \frac{-1}{4 \operatorname{Re}(\Delta \eta)^{2}}\left[\left(f_{i+1}-f_{i-1}\right)^{2}\right. \\
& \left.-4 f_{i}\left(f_{i+1}-2 f_{i}+f_{i-1}\right)\right] \\
+ & \frac{f_{i+2}-2 f_{i+1}+2 f_{i-1}-f_{i-2}}{2(\Delta \eta)^{3}} \\
+ & \frac{\alpha}{2(\Delta \eta)^{4}}\left[\left(f_{i+1}-f_{i-1}\right)\right. \\
& \left(f_{i+2}-2 f_{i+1}+2 f_{i-1}-f_{i-2}\right) \\
& -2 f_{i}\left(f_{i+2}-4 f_{i+1}+6 f_{i}-4 f_{i-1}+f_{i-2}\right)
\end{aligned}
$$




$$
\begin{aligned}
& \left.-3\left(f_{i+1}-2 f_{i}+f_{i-1}\right)^{2}\right] \\
+ & \frac{2 y}{(\Delta \eta)^{4}}\left(f_{i+1}-2 f_{i}+f_{i-1}\right)^{2} \\
+ & \frac{3 \beta \Lambda^{2}}{(\Delta \eta)^{7}}\left(f_{i+1}-2 f_{i}+f_{i-1}\right)^{2}\left(f_{i+2}-2 f_{i+1}\right. \\
& \left.+2 f_{i-1}-f_{i-2}\right) \\
+ & \Lambda K^{2} \frac{\cosh K \eta_{m}}{\cosh K} \\
\mathcal{R}_{N-1}= & \frac{-1}{4 R e(\Delta \eta)^{2}}\left[f_{N-2}^{2}+8 \Delta \eta f_{N-2}+32(\Delta \eta)^{2}\right] \\
+ & \frac{3 f_{N-2}-f_{N-3}+6 \Delta \eta}{(\Delta \eta)^{3}} \\
- & \frac{\alpha}{(\Delta \eta)^{4}}\left[f_{N-2}\left(3 f_{N-2}-f_{N-3}+6 \Delta \eta\right)\right. \\
& -2 \Delta \eta\left(6 f_{N-2}-4 f_{N-3}+f_{N-4}+8 \Delta \eta\right) \\
& \left.+3\left(f_{N-2}+4 \Delta \eta\right)^{2}\right] \\
+ & \frac{2 y}{(\Delta \eta)^{4}\left(f_{N-2}+4 \Delta \eta\right)^{2}} \\
+ & \frac{6 \beta \Lambda^{2}}{(\Delta \eta)^{7}}\left(f_{N-2}+4 \Delta \eta\right)^{2}\left(3 f_{N-2}-f_{N-3}+6 \Delta \eta\right) \\
+ & \Lambda K^{2} \frac{\cosh K \eta_{N-1}}{\cosh K}, \\
+ & \frac{6 \beta \Lambda^{2}}{(\Delta \eta)^{7}}\left(f_{N-2}+4 \Delta \eta\right)^{2}\left(3 f_{N-2}-f_{N-3}+6 \Delta \eta\right) \\
= & \frac{-f_{N-1}^{2}}{R e(\Delta \eta)^{2}}+\frac{3 f_{N-2}-f_{N-3}+6 \Delta \eta}{(\Delta \eta)^{3}}\left[4 \Delta \eta\left(-3 f_{N-2}+f_{N-3}\right)\right. \\
+ & \left.+\left(f_{N-2}+4 \Delta \eta\right)^{2}\right] \\
\mathcal{R}_{N} & \frac{2 y}{(\Delta \eta)^{4}}\left(f_{N-2}+4 \Delta \eta\right)^{2} \\
+ & \\
+ &
\end{aligned}
$$

For computation of the solution of non-linear system of algebraic equations (17), Newton's iterative method that converges quadratically, is used. In order to stabilize the convergence at an early stage of the iteration and to save computing time the damped Newton's method [30] is used which is given by

$$
\mathbf{f}^{k+1}=\mathbf{f}^{k}-\lambda^{k} J^{-1}\left(\mathbf{f}^{k}\right) \mathcal{R}\left(\mathbf{f}^{k}\right), \quad k=0,1, \ldots
$$

where $\mathbf{f}=\left[f_{1}, f_{2}, \ldots, f_{N}\right]^{T}$ represents the column vector of unknowns, $0<\lambda^{k}<1$ is the $k^{\text {th }}$ damping parameter, and $J\left(\mathbf{f}^{k}\right)$ is the Jacobian matrix evaluated at the $k^{\text {th }}$ iter- ate. Here the Jacobian matrix $J(\mathbf{f})$ is

$$
\left[\frac{\partial \mathcal{R}_{j}}{\partial f_{i}}\right]_{i, j=1,2, \ldots, N}=\left[\begin{array}{ccccc}
\frac{\partial \mathcal{R}_{1}}{\partial f_{1}} & \frac{\partial \mathcal{R}_{1}}{\partial f_{2}} & \frac{\partial \mathcal{R}_{1}}{\partial f_{3}} & \ldots & \frac{\partial \mathcal{R}_{1}}{\partial f_{N}} \\
\frac{\partial \mathcal{R}_{2}}{\partial f_{1}} & \frac{\partial \mathcal{R}_{2}}{\partial f_{2}} & \frac{\partial \mathcal{R}_{2}}{\partial f_{3}} & \ldots & \frac{\partial \mathcal{R}_{2}}{\partial f_{N}} \\
\vdots & \vdots & \vdots & \ddots & \vdots \\
\frac{\partial \mathcal{R}_{N}}{\partial f_{1}} & \frac{\partial \mathcal{R}_{N}}{\partial f_{2}} & \frac{\partial \mathcal{R}_{N}}{\partial f_{3}} & \cdots & \frac{\partial \mathcal{R}_{N}}{\partial f_{N}}
\end{array}\right] .
$$

A good initial guess is necessary for the convergence of the damped Newton's method and very important for the fast solution of the iterative process. The initial solution guess for the axial velocity is approximated according to the boundary conditions. For this problem the convergence of the damped Newton method is achieved when

$$
\left\|J^{-1}\left(\mathbf{f}^{k+1}\right)\right\|_{2}-\left\|J^{-1}\left(\mathbf{f}^{k}\right)\right\|_{2}<10^{-4}, \quad k=0,1, \ldots
$$

\section{Results and Discussion}

The result of our investigation is discussed in this section with graphical presentation of $f^{\prime}(\eta)$ and $f(\eta)$ which correspond to the axial velocity and vertical velocity, for various governing flow parameters. The effect of the visco-elastic parameter $\alpha$, the cross viscous parameter $y$, the third grade material parameter $\beta$, the Reynold's number $R e$ and the Debye Hückel parameter $K$ on the velocity profiles is studied through numerical comparisons among the relevant curves. Although computation for several combinations of flow parameters were performed, only some key graphs are provided in this paper.

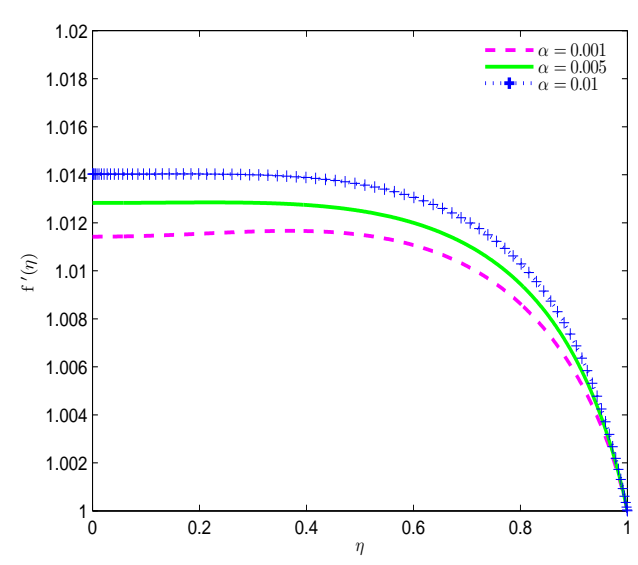

Fig. 2: Variation of $f^{\prime}$ with $\alpha$ at $\beta=0.005, y=0.01, R e=$ $10.0, K=10.0$ and $\Lambda=0.001$.

Figures 2-6 illustrate the shape of the dimensionless axial velocity profile for different flow parameters. It is 


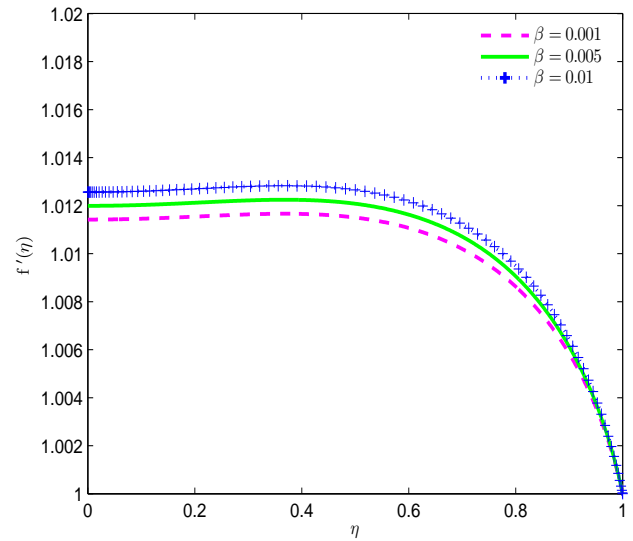

Fig. 3: Variation of $f^{\prime}$ with $\beta$ at $\alpha=0.01, y=0.01, R e=10.0$, $K=10.0$ and $\Lambda=0.001$.

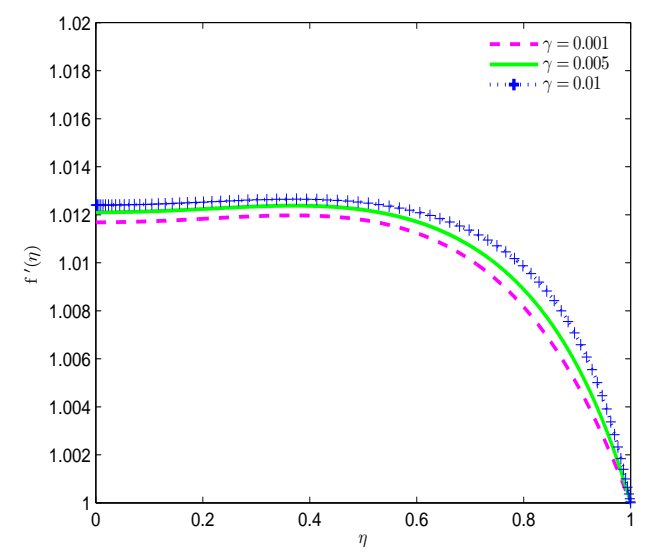

Fig. 4: Variation of $f^{\prime}$ with $y$ at $\alpha=0.01, \beta=0.005, R e=$ $10.0, K=10.0$ and $\Lambda=0.001$.

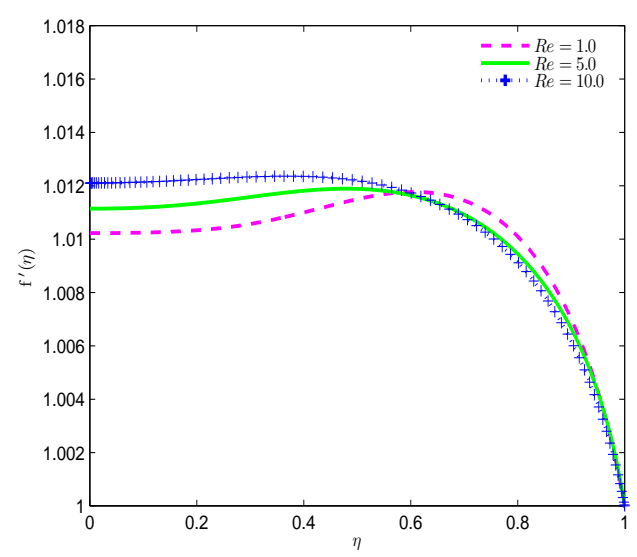

Fig. 5: Variation of $f^{\prime}$ with $\operatorname{Re}$ at $\alpha=0.01, \beta=0.005, y=0.01$, $K=10.0$ and $\Lambda=0.001$. depicted from Fig.2 that the axial velocity increases for increasing viscoelastic parameter, $\alpha$. The velocity profile maintains a constant value upto a certain distance from the centreline and thereafter decreases monotonically as approaches the wall. Similar behavior is observed for the case of varying $\beta$, the third grade material parameter and $y$, the cross viscous parameter as shown in Fig. 3 and Fig. 4 , respectively. Variation of the axial velocity for various $R e$ is provided in Fig. 5. The velocity increases for increasing value of $R e$ upto $\eta=0.6$ and for $\eta>0.6$ the behavior of the velocity gets reversed for increasing $R e$. Further $R e$ does not have much effect on $f^{\prime}$ near the boundary for $R e>5$.0. Fig. 6 shows the variation of horizontal component of velocity for different values of the Debye-Hückel parameter, $K$. As $K$ increases $f^{\prime}$ increases but a deviation is observed very close to the channel wall which is displayed in the inset figure.

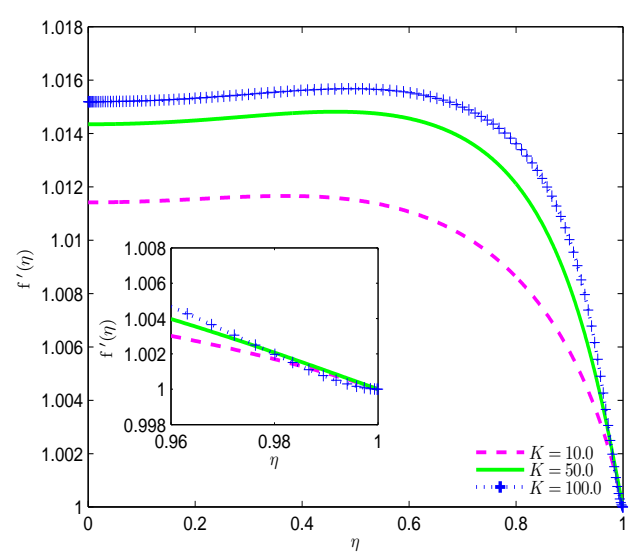

Fig. 6: Variation of $f^{\prime}$ with $K$ at $\alpha=0.01, \beta=0.005, y=0.01$, $R e=10.0$ and $\Lambda=0.001$.

Figures 7-11 represent vertical velocity profiles for different flow parameters. It appears to increase from zero on the axis while moving away from it and finally approaches zero again at the boundary. The velocity increases with increasing values of $\alpha, \beta, y, K$ and $R e$. The figures show that these flow parameters do not affect the velocity near the channel wall. The shape of the vertical velocity curve changes for $R e>5$.0. It can be estimated quantitavely that the magnitude of the vertical velocity component is lesser than the axial velocity component by comparing the results presented in both flow cases.

The figures presenting the velocity profile reveal the fact that velocity increases with the rise in non-Newtonian parameters $\alpha, y$ and $\beta$ which is physically relevant. As the visco-elastic parameter $\alpha$ and cross viscous param- 


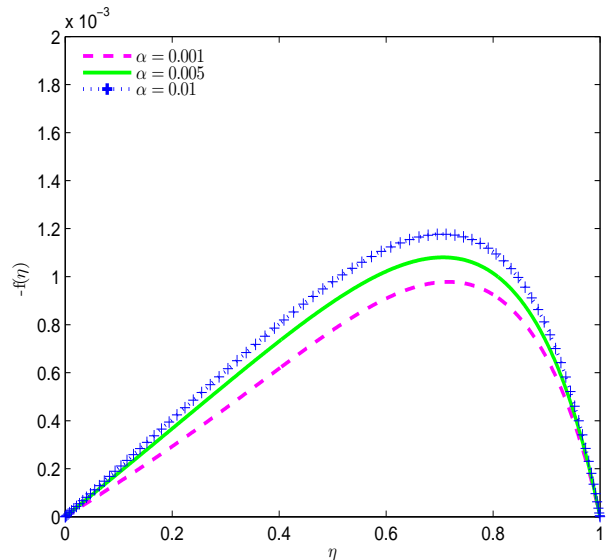

Fig. 7: Variation of $f$ with $\alpha$ at $\beta=0.005, y=0.01, R e=10.0$, $K=10.0$ and $\Lambda=0.001$.

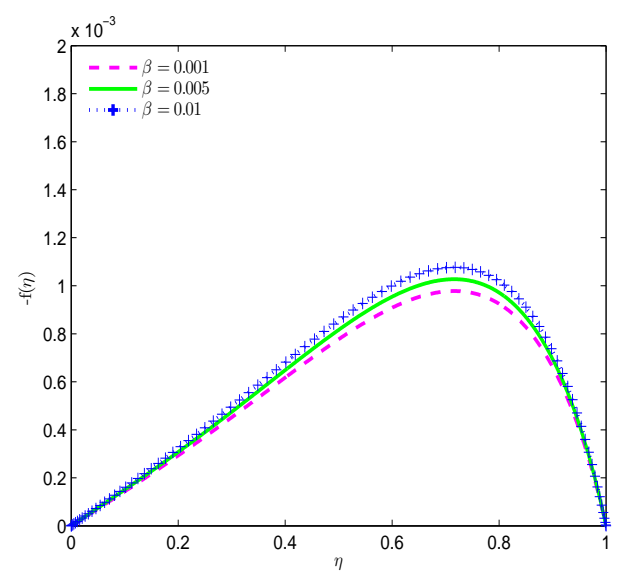

Fig. 8: Variation of $f$ with $\beta$ at $\alpha=0.01, y=0.01, R e=10.0$, $K=10.0$ and $\Lambda=0.001$.

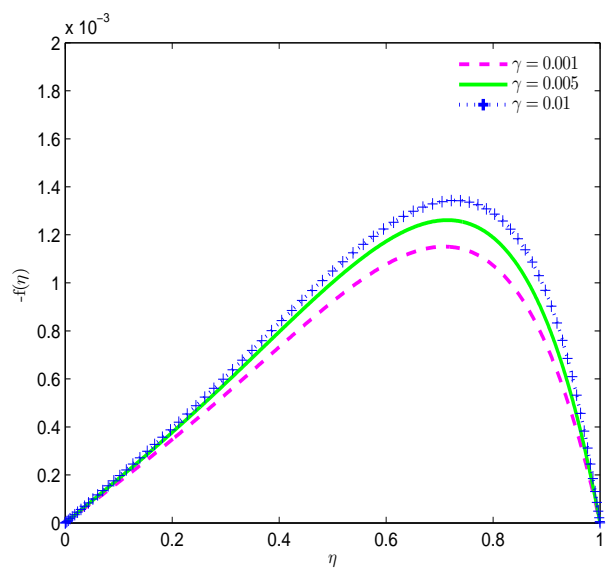

Fig. 9: Variation of $f$ with $y$ at $\alpha=0.01, \beta=0.005, R e=10.0$, $K=10.0$ and $\Lambda=0.001$.

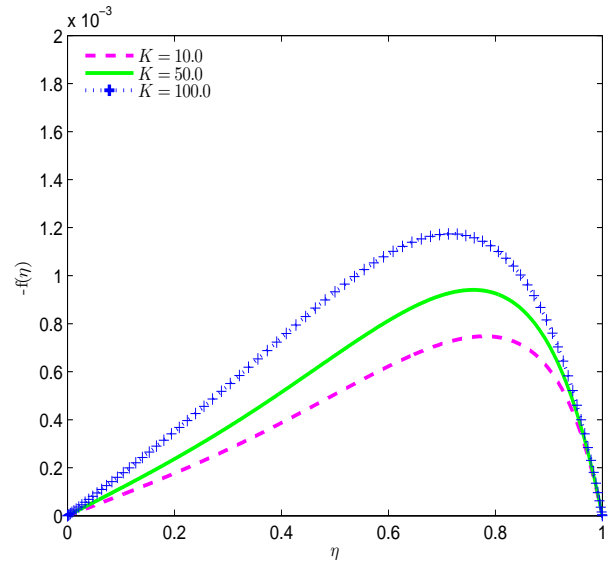

Fig. 10: Variation of $f$ with $K$ at $\alpha=0.01, \beta=0.005, y=0.01$, $R e=10.0$ and $\Lambda=0.001$.

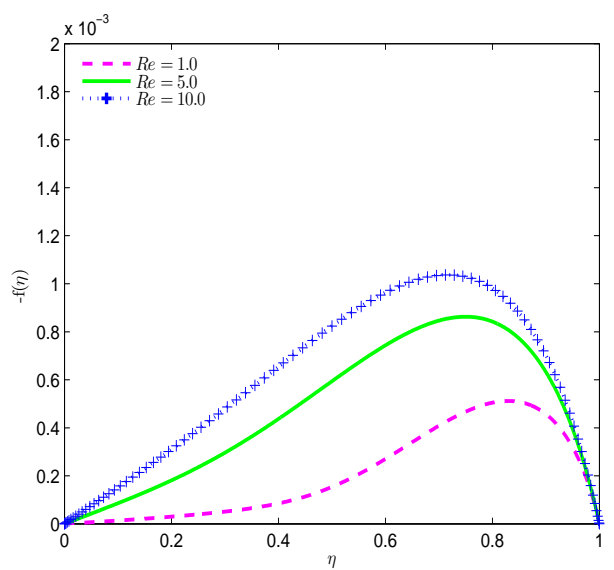

Fig. 11: Variation of $f$ with $\operatorname{Re}$ at $\alpha=0.01, \beta=0.005, y=0.01$, $K=10.0$ and $\Lambda=0.001$.

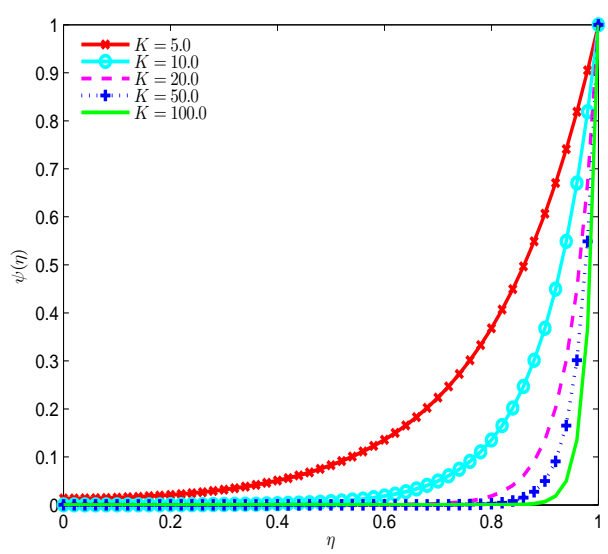

Fig. 12: Variation of electric potential distribution $\psi$ with $K$. 
eter $y$ increases, the shear rate increases leading to increase in the deformation, rearrangement and aggregation of fluid particles, thereby increasing the fluid velocity in the channel. Similarly, with increasing shear thinning parameter $\beta$ the viscosity of the fluid decreases, resulting in the increase of velocity. The figures associated with the Reynold's number $R e$ show that the inertial forces dominate the flow in the middle of the channel whereas near the wall viscous forces dominate and this is due to the presence of the electric double layer. The Debye Hückel parameter is directly proportional to the width of the channel and inversely proportional to the Debye length, which describes the physical extent of the EDL. It is observed that the velocity increases with increasing width of the channel but simultaneously, the effect of EDL is noticed at the walls of the channel.

The potential distribution function $\psi$ has been plotted against $\eta$ for different values of $K$ in Fig. 12. The value of $\psi$ is zero at the middle of the channel and for $K>20.0$ it increases sharply to attain maximum on the boundary. It can also be inferred that as $K$ increases, the potential distribution function decreases. For $K>100.0$ variation among the curves becomes negligible. This result depicts the influence of Debye length on the potential distribution. As the EDL thickness reduces, the potential distribution function decreases and beyond a certain $\lambda_{D}$ the potential distribution function $\psi$ starts behaving similarly with negligible variation.

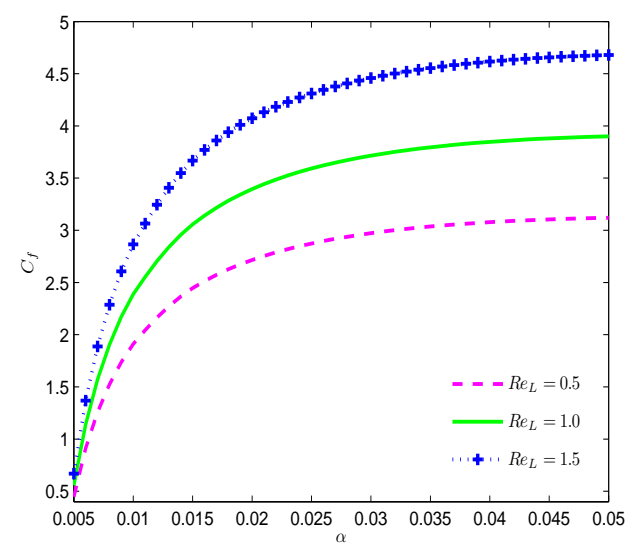

Fig. 13: Variation of $C_{f}$ with $\alpha$ for $\beta=0.005$.

In addition, we have computed the local skin-friction coefficient as

$$
C_{f}=\left.\frac{\tau_{x y}}{\rho u^{2}}\right|_{y=R_{0}},
$$

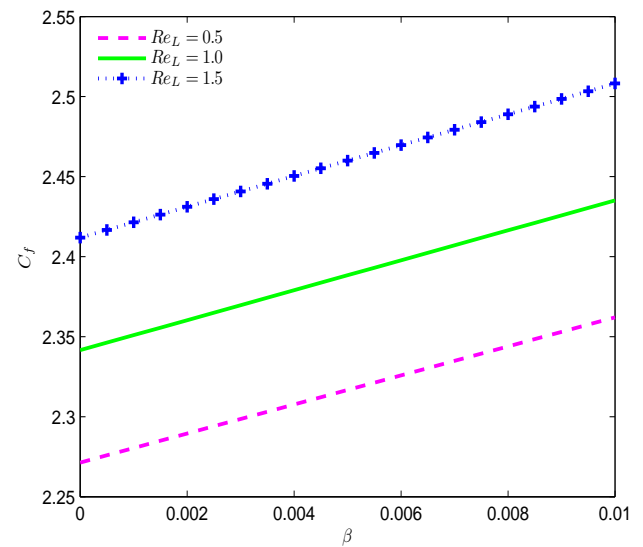

Fig. 14: Variation of $C_{f}$ with $\beta$ for $\alpha=0.001$.

which in terms of dimensionless quantities become

$$
\begin{aligned}
\operatorname{Re}_{L} C_{f} & =f^{\prime \prime}(1)+\alpha\left[1-2 f^{\prime \prime}(1)\right] \\
& +2 \beta\left[2 f^{\prime \prime}(1)+f^{\prime \prime 3}(1)\right],
\end{aligned}
$$

where $R e_{L}=\frac{b R_{0}^{2} \rho}{\mu}$ is the local Reynold's number. It is observed that the skin-friction coefficient increases with increasing viscoelastic parameter, $\alpha$, and increases linearly with increasing shear-thinning parameter, $\beta$ from Fig. 13 and Fig. 14, respectively. Further, $b$, the stretching parameter is directly proportional to the local Reynold's number and it is evident from the figures that increase in the stretching parameter results in increasing value of coefficient of skin friction.

\section{Conclusion}

In the present paper, the electro-osmotic flow of a third grade fluid in a channel having stretching walls is investigated with a view to apply it in the study of blood flow dynamics. The non-linear differential equation is discretized by finite difference method and the resulting system of non-linear algebraic equations is solved by the damped Newton's method. The results computed are presented graphically for various parameters of interest. The results obtained from this study are summarized below

1. Axial velocity profiles are found to be diminishing from their maxima at the middle of the channel to achieve the minimum value on the wall surface.

2. Axial velocity and vertical velocity increases with increasing visco-elastic parameter, $\alpha$, cross viscous parameter, $y$ and increasing third grade fluid parameter, $\beta$. 
3. Axial velocity increases with the increasing DebyeHückel parameter, $K$, but near the wall reverse of it is observed.

4. Potential distribution function, $\psi$ is minimum at the middle of the channel and gradually increases as it approaches the wall. It decreases with increasing Debye-Hückel parameter, $K$.

5. Value of local skin-friction coefficient, $C_{f}$ increases with increasing values of visco-elastic parameter, $\alpha$, third grade fluid material parameter, $\beta$, and the stretching parameter $b$.

\section{References}

[1] C.M. Ho, Y.C. Tai, “Micro-Electro-Mechanical-Systems (MEMS) and fluid flows”, Annual Review of Fluid Mechanics, 30, 579612, 1998

[2] H.A. Stone, A.D. Stroock, A. Ajdari, “ Engineering flows in small devices:microfluidics toward a lab-on-a-chip”, Annual Review of Fluid Mechanics, 36, 381-411, 2004

[3] F.F. Reuss, "Sur un nouvel effet de l'electricite galvanique", Memoires de la Societe Imperiale des Naturalistes de Moscou, 2, 327-337, 1809

[4] W.T. Hanna, J.F. Osterle, "Transient electro-osmosis in capillary tubes”, The Journal of Chemical Physics, 49, 4062-4068, 1968

[5] C.F. Ivory, "Transient electroosmosis: The momentum transfer coefficient”, Journal of Colloid and Interface Science, 96, 296298, 1983

[6] H.J. Keh, H.C. Tseng, "Transient electrokinetic flow in fine capillaries", Journal of Colloid and Interface Science, 242, 450459, 2001

[7] J.G. Santiago, "Electroosmotic flows in microchannels with finite inertial and pressure forces", Analytical Chemistry, 73, 2353-2365, 2001

[8] Y. Kang, C. Yang, X. Huang, "Dynamic aspects of electroosmotic flow in a cylindrical microcapillary", International Journal of Engineering Science, 40, 2203-2221, 2002

[9] S.Chakraborty, "Electroosmotically driven capillary transport of typical non-Newtonian biofluids in rectangular microchannels”, Analytica Chimica Acta, 605, 175-184, 2007

[10] H.M. Park, W.M. Lee, "Effect of viscoelasticity on the flow pattern and the volumetric flow in electroosmotic flows through a microchannel", Lab on a chip, 8, 1163-1170, 2008

[11] C.L.A. Berli, M.L. Olivares, "Electrokinetic flow of nonNewtonian fluids in microchannels", Journal of Colloid and Interface Science, 320, 582-589, 2008

[12] C. Zhao, E. Zholkovskij, J.H. Masliyah, C. Yang, "Analysis of electroosmotic flow of power-law fluids in a slit microchannel”, Journal of Colloid and Interface Science, 326, 503-510, 2008

[13] A.A. Siddiqui, A. Lakhatia, "Steady electro-osmotic flow of a micropolar fluid in a microchannel", Proceedings of the Royal Society A, 465, 501-522, 2009
[14] G.H. Tang, X.F. Li, Y.L. He, W.Q. Tao, “Electro-osmotic flow of non-Newtonian fluid in microchannels", Journal of NonNewtonian Fluid Mechanics, 157, 133-137, 2009

[15] A.M. Afonso, M.A. Alves, F.T. Pinho, "Analytical solution of mixed electro-osmotic pressure driven visco-elastic fluids in microchannels", Journal of Non-Newtonian Fluid Mechanics, 159, 50-63, 2009

[16] N. Vasu, S. De, "Electroosmotic flow of a power-law fluids at high zeta potentials”, Colloids and surfaces A: Physiochemical and Engineering Aspects, 368, 44-52, 2010

[17] M.Hadigol, R. Nosrati, A. Nourbakhsh. M. Raisee, "Numerical study of electroosmotic micromixing of non-Newtonian fluids", Journal of Non-Newtonian Fluid Mechanics, 166, 965-971, 2011

[18] A.Sadeghi, M.H. Saidi, A.A. Mozafari, "Heat transfer due to electroosmotic flow of viscoelastic fluids in a slit microchannel”, International Journal of Heat and Mass Transfer, 54, 4069-4077, 2011

[19] Y. Jian, J. Su, L. Chang, Q. Liu, G. He, "Transient electroosmotic flow of general Maxwell fluids through a slit microchannel", Mathematical Physics, 65, 435-447, 2014

[20] A.J. Moghadam, P. Akbarzadeh, "Time-periodic electroosmotic flow of non-Newtonian fluids in microchannels", International Journal of Engineering, 29, 706-714, 2016

[21] E. Jimenez, J. Escandon, O. Bautista, F. Mendez, "Start-up electroosmotic flow of Maxwell fluids in a rectangular microchannel with high zeta potentials", Journal of Non-Newtonian Fluid Mechanics, 227, 17-29, 2016

[22] Z. Xie, Y. Jian, "Rotating electroosmotic flow of power-law fluids at high zeta potentials", Colloids and surfaces A: Physicochemical and Engineering Aspects, 461, 231-239, 2014

[23] H. Yoshida, T. Kinjo, H. Washizu, “Analysis of electro-osmotic flow in a microchannel with undulated surfaces”, Computers and Fluids, 124, 237-245, 2016

[24] C. Qi, C. Ng, "Electroosmotic flow of a power-law fluid in a slit microchannel with gradually varying channel height and wall potential”, European Journal of Mechanics-B/Fluids, 52, 160-168, 2015

[25] B. Srinivas, "Electroosmotic flow of a power-law fluid in an elliptic microchannel”, Colloids and surfaces A: Physicochemical and Engineering Aspects, 492, 144-151, 2016

[26] J.C. Misra, G.C. Shit, S. Chandra, P.K. Kundu, “Electro-osmotic flow of a visco-elastic fluid in a channel:Applications to physiological fluid mechanics", Applied Mathematics and Computation, 217, 7932-7939, 2011

[27] R.L. Fosdick, K.R. Rajagopal, “Thermodynamics and stability of fluids of third grade", Proceedings of the Royal Society A, 369(1738), 351-377, 1980

[28] H. Bruss, "Theoretical Microfluidics", Oxford Master Series in Condensed Matter Physics, Oxford University Press, Oxford, UK, 2008

[29] M. Pakdemirli, "The boundary layer equations of third grade fluids”, International Journal of Non-linear Mechanics, 27, 785793, 1992

[30] S.D. Conte, Carl de Boor, "Elementary Numerical Analysis: An Algorithmic Approach", 2nd edition, McGraw-Hill, New York, 1972 